\title{
Edge atoms effects on the perpendicular anisotropy of ultrathin magnetic layers
}

J. Geshev, A. Gündel, I. Zaharieva, and J. E. Schmidt

Citation: Appl. Phys. Lett. 101, 132407 (2012); doi: 10.1063/1.4754621

View online: http://dx.doi.org/10.1063/1.4754621

View Table of Contents: http://aip.scitation.org/toc/apl/101/13

Published by the American Institute of Physics

\section{A|P | Applied Physics AlP| Letters}

Save your money for your research.

It's now FREE to publish with us no page, color or publication charges apply. 


\title{
Edge atoms effects on the perpendicular anisotropy of ultrathin magnetic layers
}

\author{
J. Geshev, ${ }^{1}$ A. Gündel, ${ }^{1}{ }^{12}$ I. Zaharieva ${ }^{3}$ and J. E. Schmidt ${ }^{1}$ \\ ${ }^{1}$ Instituto de Física, UFRGS, 91501-970 Porto Alegre, RS, Brazil \\ ${ }^{2}$ Universidade Federal do Pampa, Campus Bagé, 96413-170 Bagé, RS, Brazil \\ ${ }^{3}$ Freie Universität Berlin, Fachbereich Physik, Arnimallee 14, D-14195 Berlin, Germany
}

(Received 30 March 2012; accepted 10 September 2012; published online 25 September 2012)

The present work reports experimental and theoretical results for electrodeposited $\mathrm{Co} / \mathrm{Au}(111)$ ultrathin layers with very specific magnetic behavior. We show that the observed two peaks in the out-of-plane magnetization versus deposition time variation could be explained by the remarkably high perpendicular anisotropy of the perimeter atoms of low-dimensional islands formed during the layer-by-layer growth, as compared to that of the surface atoms. Our results indicate that it is possible to sustain high anisotropy in very small grains without coming across the superparamagnetic limit, opening excellent opportunities for materials engineering. (C) 2012 American Institute of Physics. [http://dx.doi.org/10.1063/1.4754621]

Magnetic anisotropy (MA) is one of the key characteristics of ferromagnetic nanostructures which, due to their reduced dimensionality, may have properties very different from those observed in bulk materials. ${ }^{1}$ Néel suggested ${ }^{2}$ that reduced symmetry gives rise to surface/interface MA with magnitude $K_{S}$, independent of the film's thickness. This theory, however, cannot be readily applied in the limit of several atomic layers where a separation of the sample into bulk and two surfaces does not seem to be appropriate. ${ }^{3}$ Experiments have shown that free atoms and twodimensional (2D) clusters develop giant MA and enhanced magnetic moment due to the decreased effective coordination number and the increased electron localization. ${ }^{4-9}$

Perpendicular $(\perp)$ MA has been observed in Fe and Co films electrodeposited onto $\mathrm{Au}(111) .^{10,12-14}$ Two peaks in the out-of-plane magnetization, $M_{\perp}$, versus deposition time, $t$ (or thickness, $d$ ), curves have been observed for Co while Fe samples show a single peak only. The later and the first peak for the Co films have been attributed to the competition between demagnetization and Néel type anisotropies. ${ }^{10,12,14}$ No explanation, however, has been given for the second peak that diminishes and vanishes when the value of the external magnetic field, $H$, applied during the film growth, is decreased. Remarkably, Co deposition on $\mathrm{Au}(111)$ starts with a fast nucleation of two monolayer (ML) high crystallites with facecentered cubic (fcc) crystal structure followed by lateral growth with increasing coverage. After the completion of the first bilayer, a layer-by-layer growth is observed at least up to 5 MLs, accompanied by fcc-hcp(0001) transformation (hcp, hexagonal close packing). ${ }^{4,13,15}$

The present work shows that both peaks in $M_{\perp}(t)$ of electrodeposited $\mathrm{Co} / \mathrm{Au}(111)$ films could be solely attributed to the very high (up to four times the respective bulk value) $\mathrm{MA}$ of the edge atoms of $2 \mathrm{D}$ islands formed during growth as compared to that of the surface atoms.

Cobalt layers were electrodeposited on $100 \mathrm{~nm}$ thick gold films evaporated on freshly cleaved mica substrates. The substrates were flame annealed before use to obtain the 22 $\times \sqrt{3}$ surface reconstruction and large (111) textured singlecrystalline grains. ${ }^{10,13}$ Electrochemical experiments were con- ducted with a classical three-electrode cell under potentiostatic mode using a saturated $\mathrm{Hg} / \mathrm{Hg}_{2} \mathrm{SO}_{4}$ as a reference electrode. The solution consisted of $10 \mathrm{mM} \mathrm{K}_{2} \mathrm{SO}_{4}+0.1 \mathrm{mM} \mathrm{KCl}$ $+1 \mathrm{mM} \mathrm{H}_{2} \mathrm{SO}_{4}+1 \mathrm{mM} \mathrm{CoSO}_{4}$. More details on the in situ techniques employed for structural [home-built scanning tunneling microcopy (STM)], and magnetic (home-built alternating gradient field magnetometer) characterizations can be found in previous works. ${ }^{10,16}$

Room temperature $M_{\perp}(t)$ transients, recorded in situ during Co deposition at different $U$ and in constant value of $H$, are shown in Fig. 1(a). It is seen that varying $U$ modifies the time-scale because of a variation of the deposition rate with $U$. The curves for $|U| \leq 1.4 \mathrm{~V}$ are characterized by two distinct maxima, in contrast to those for deposition at higher $|U|$, where the unique maximum is wider. Figure 1(b) shows $M_{\perp}(t)$ curves measured at various fields for fixed $U$. It is worth emphasizing that the Co growth rate depends not only

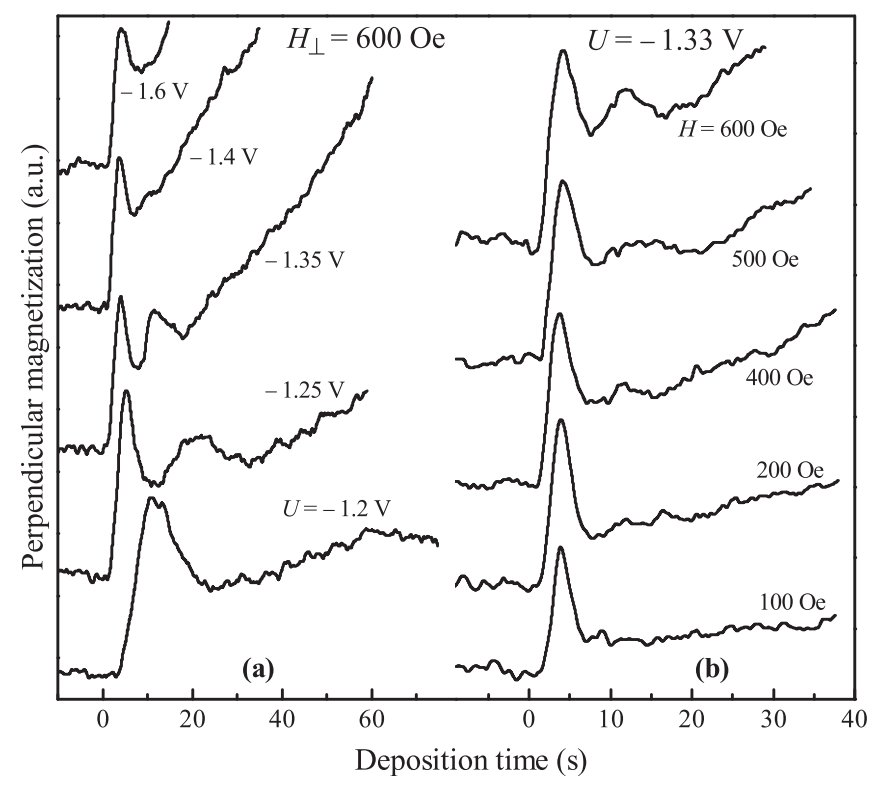

FIG. 1. $M_{\perp}(t)$ transients recorded in situ during Co electrodeposition using EC1 (a) varying $U$ for fixed $H=600$ Oe or (b) varying $H$ for constant $U=-1.33 \mathrm{~V}$. 
on the concentrations of the solutions and on $U$ but also on the configuration of electrodes inside the particular electrochemical cell used. ${ }^{17}$ The experimental data presented in Figs. 1, 3(d), and 3(e) were obtained using an electrochemical cell (denoted as EC1), which had different electrode configuration than the cell used in the experiment presented in Fig. 3(c) (referred to as EC2). We verified that despite the different deposition rates of EC1 and EC2, the shape of the transients and the Co thicknesses corresponding to the extrema of $M_{\perp}(t)$ remain the same regardless the cell used. Note that it is impossible to exactly reproduce any $M(t)$ since the preparation and deposition conditions of distinct pieces of samples cannot be exactly the same, e.g., slightly different flame annealing of the substrate may lead to distinct growth rates.

We focus our attention on one representative Co sample deposited at $U=-1.3 \mathrm{~V}$ using EC2, which $M_{\perp}(t)$ and $M_{\|}(t)$ (the projections of the saturation magnetization along the direction of $\mathbf{H}$ when it is applied perpendicular to or in the plane of the films, respectively, where the symbol $\|$ refers to in-plane $\mathbf{H})$ variations are shown in Fig. 3(c). $M_{\perp}(t)$ reaches the first maximum at $t \approx 4 \mathrm{~s}$ and the second, less pronounced one, at $t \approx 28 \mathrm{~s}$. Finally, after a minimum at $t \approx 40 \mathrm{~s}$, an approximately linear regime is observed; the two minima correspond to $d \approx 2$ and $3 \mathrm{MLs}$, respectively. The cobalt layers' thicknesses were estimated by using the stripping method. ${ }^{10}$ Our real-time in situ STM observations for $U=-1.3 \mathrm{~V}$ confirmed that the first Co layer is biatomic and the following Co layers' growth is, at least until the fifth atomic plane, a quasi perfect ML by ML one, in agreement with previous observations. ${ }^{13}$ Growth rate of the first Co bilayer approximately four times larger than that of the subsequent atomic layers has already been observed previously, ${ }^{11}$ the same trend being confirmed for all values of $U$. STM images have indicated the presence of residual strain of $\approx 4 \%$ progressively reduced upon further deposition, ${ }^{13}$ and it has been speculated that strain relief mechanisms that involve $\mathrm{Au} / \mathrm{Co}$ intermixing at the interface could be responsible for the above growth rate difference. No peaks were obtained in $M_{\|}(t)$ for all $U$ and $H$.

We performed a series of simulations trying to reproduce this type of magnetic behavior considering a number of sets of conventionally used parameters, including a cubic MA constant (characteristic for nanosized fcc Co) and/or both first- and second-order uniaxial (hcp) ones for 3 and more MLs as well as variable demagnetization factor. ${ }^{4,18}$ All attempts to reproduce the second peak using classical 2D models failed unless a non-monotonic $K_{S} / d$ is considered which, however, does not seem to be physically justified since the islands' height is constant, 2 and 3 MLs during the growth of the first bilayer and of the third ML, respectively. The competition between $K_{S} / d$ and demagnetization (shape) terms cannot be responsible for the peaks, even considering thickness- and geometry-dependent demagnetization factor, an effect that may come into play for rather large ML/bilayer platelets of a few hundred atoms in diameter. ${ }^{18}$

Thus, we changed our approach based also on some important reports ${ }^{6,7}$ where it has been shown that atoms at the edge of the Co islands exhibit a much higher MA than atoms from inside the islands, which is largely independent of the perimeter shape and crystallographic orientation, in accordance with theoretical predictions. ${ }^{19}$ We estimated the contributions to the total magnetization of the atoms at the perimeter (AP) and atoms at the surface (AS), with respective numbers $N_{\mathrm{AP}}$ and $N_{\mathrm{AS}}$, as well as their deposition time dependence. Consider a layer formed by laterally growing and/or coalescing islands with magnetization $M(t)=N_{\mathrm{AP}}(t)$ $m_{\mathrm{AP}}(t)+N_{\mathrm{AS}}(t) m_{\mathrm{AS}}(t)$, where the two terms are the contributions of AP and AS with normalized (to their saturation values, $M_{s}$ ) magnetizations $\mathbf{m}_{\mathrm{AP}}$ and $\mathbf{m}_{\mathrm{AS}}$, respectively. $m_{\mathrm{AP}(\mathrm{AS})}$ at each $t$ are obtained by minimizing the respective free magnetic energy per unit volume, $E$,

$$
\frac{E}{M_{S}}=\frac{K_{\mathrm{eff}}^{\mathrm{AP}(\mathrm{AS})}}{M_{S}} \cos ^{2} \theta_{\mathrm{AP}(\mathrm{AS})}-\mathbf{H} \cdot \mathbf{m}_{\mathrm{AP}(\mathrm{AS})}
$$

in respect to $\theta_{\mathrm{AP}(\mathrm{AS})}$, the angles between $\mathbf{m}_{\mathrm{AP}(\mathrm{AS})}$ and the normal-to-the-plane direction. Free parameters are the effective MA constants $K_{\mathrm{eff}}^{\mathrm{AP}}$ (AS) which may consist of demagnetization $\left(K_{D}\right)$, intrinsic $\left(K_{1}\right)$, and surface $\left(K_{S} / d\right)$ contributions. For $K_{\text {eff }}<0$, the easy axis is perpendicular to the plane; otherwise, the MA is an easy plane one. For system's $z$-axis parallel to [111], one obtains $E_{c}=K_{1}^{\mathrm{fcc}}\left[\frac{\sqrt{2}}{3} \sin ^{3} \theta \cos \theta \sin \phi\right.$ $\left.\left(1-4 \cos ^{2} \phi\right)+\frac{1}{4} \sin ^{4} \theta+\frac{1}{3} \cos ^{4} \theta\right]$ being $\phi$ the azimuthal angle of $\mathbf{m}_{\mathrm{AP}}$ (or $\mathbf{m}_{\mathrm{AS}}$ ). Since the value of the intrinsic cubic MA, $E_{c}$, of fcc Co atoms yield using ${ }^{20} K_{1}^{\mathrm{fcc}}=-0.8$ $\times 10^{6} \mathrm{erg} / \mathrm{cm}^{3}$ is negligible as compared to the $\left(K_{S} / d\right) \cos ^{2} \theta$ energy estimated here, this MA has been omitted.

There are two possible solutions for the configurations considered: $m_{\mathrm{AP}(\mathrm{AS})}=H / H_{\mathrm{eff}}$ if $\mathbf{H}$ is $\perp$ to the easy axis (or to the easy plane, respectively) for $H<H_{\mathrm{eff}}\left[=2 K_{\mathrm{eff}}^{\mathrm{AP}(\mathrm{AS})}\right.$ $/ M_{s}$, the effective MA field]; otherwise $m_{\mathrm{AP}(\mathrm{AS})}=1$, which is also valid for $\mathbf{H}$ along the easy axis (or in the easy plane). The expressions for $M_{\perp}(t)$ resulting from the minimizations are

$$
\begin{gathered}
M_{\perp, \mathrm{A}}=2 N_{\mathrm{AP}}+2 N_{\mathrm{AS}} \frac{H}{H_{\mathrm{eff}}^{\mathrm{AS}, \mathrm{fcc}}}, \\
M_{\perp, \mathrm{B}}=N_{\mathrm{AP}} \frac{H}{H_{\mathrm{eff}}^{\mathrm{AP}, \mathrm{B}}}+2\left(L^{2}-N_{\mathrm{AS}}\right) \frac{H}{H_{\mathrm{eff}}^{\mathrm{AS}, \mathrm{fcc}}} \\
+3 N_{\mathrm{AS}} \frac{H}{H_{\mathrm{eff}}^{\mathrm{AS}, \mathrm{hcp}}}, \\
M_{\perp, \mathrm{C}}=N_{\mathrm{AP}} \frac{H}{H_{\mathrm{eff}}^{\mathrm{AP}, \mathrm{C}}}+\left(3 L^{2}+N_{\mathrm{AS}}\right) \frac{H}{H_{\mathrm{eff}}^{\mathrm{AS}, \mathrm{hcp}}} .
\end{gathered}
$$

The corresponding expressions for $M_{\|}(t)$ are

$$
\begin{gathered}
M_{\|, \mathrm{A}}=2 N_{\mathrm{AP}} \frac{H}{H_{\mathrm{eff}}^{\mathrm{AP}, \mathrm{A}}}+2 N_{\mathrm{AS}}, \\
M_{\|, \mathrm{B}}=N_{\mathrm{AP}}+2 L^{2}+N_{\mathrm{AS}}, \\
M_{\|, \mathrm{C}}=N_{\mathrm{AP}}+3 L^{2}+N_{\mathrm{AS}} .
\end{gathered}
$$

The fcc and hcp indexes reflect the fact that Co is fcc if $d \leq 2$ MLs since at least 3 MLs are necessary for hcp structure to be formed. 
For determination of $N_{\mathrm{AP}}(t)$ and $N_{\mathrm{AS}}(t)$, we developed an algorithm that analyzes the isothermal growth of randomly oriented isotropic (circular) or anisotropic (elliptical) particles. The nucleation and 2D growth were allowed to occur in a rectangular grid with uniform mesh spacing $\Delta x$ covering an $L \times L$ area with mesh size $L$. The linear growth was considered when the new fraction obeys the Kolmogorov-Johnson-Mehl-Avrami (KJMA) kinetics, ${ }^{21-23}$ i.e., it should be $\propto 1-\exp \left(-k t^{n}\right)$, where $k$ is a nucleation- and growth-dependent constant and $n$ is the so-called Avrami's exponent. When all nuclei are present and start to grow at the beginning of the 2D transformation, $n=2$ and the nucleation is termed site saturation. If new nuclei appear at every transformation step (continuous nucleation), $n=3$.

Here the site-saturation mode was adopted, where the nucleation was assumed to happen instantaneously at the beginning. The positions and orientations of the new nuclei were chosen by using a random-number generator; STM images ${ }^{11}$ inspired the choice of $L$ (here $L=300$ corresponds to $30 \mathrm{~nm}$ ) and of the ratio between the initial number of nuclei, $N_{0}$, and $L$. It was also allowed the grains' rims, contributing to $N_{\mathrm{AP}}$, to be a several $\Delta x$ wide. At each time step, $N_{\mathrm{AP}}$ and $N_{\mathrm{AS}}$ were determined. Their average values for 1000 trials conducted (we estimated that even $\approx 200$ trials give practically the same results) were then calculated. Figure 2 shows a series of snapshots for elliptical grains with eccentricity, $\epsilon$, equal to 0.6 where, for better visualization, their rims were taken to be $L / 40$ wide. The resulting $N_{\mathrm{AP}}(t / \tau)$ and $N_{\mathrm{AS}}(t / \tau)$ are also shown, where $\tau$ is the total transformation time.

We carried out numerous calculations of $N_{\mathrm{AP}}(t)$ and $N_{\mathrm{AS}}(t)$ varying $\epsilon, N_{0}$, and $L$ for $\Delta x=1$ and observed that each $N_{\mathrm{AP}}(t)$ is characterized by a well-defined broad peak with maximum at the inflection point of the respective $N_{\mathrm{AS}}(t)$. In order to check the validity of our simulations, the so-called Avrami plots, i.e., $\ln \left[-\ln \left(1-N_{\mathrm{AS}} / L^{2}\right)\right]$ vs. $\ln (t / \tau)$,

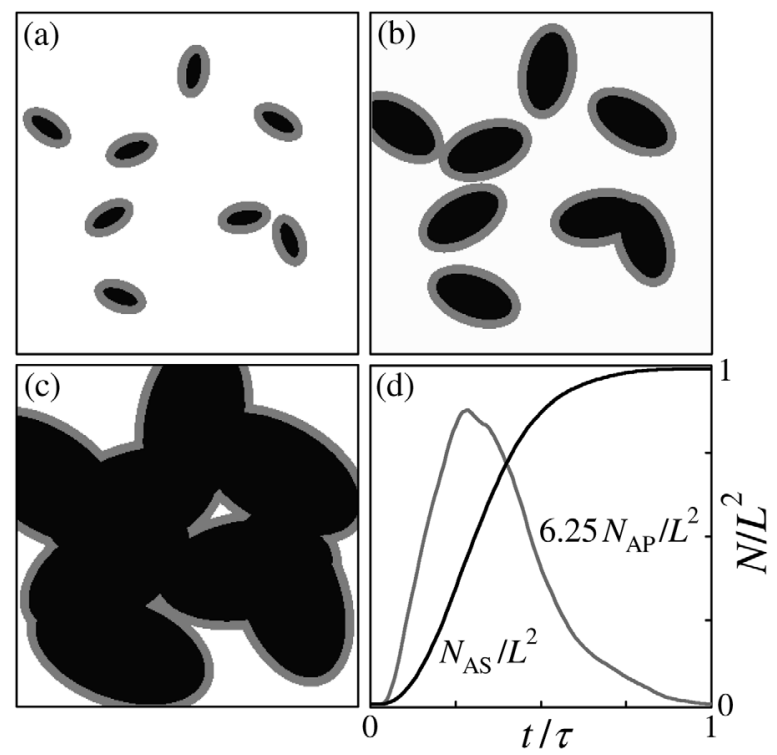

FIG. 2. (a)-(c) Snapshots of a simulation for site-saturated nucleation and isotropic growth of 8 elliptical grains with $\epsilon=0.6$ and rims with thickness $L / 40$ for $L=320$. Black and gray tones denote AS and AP, respectively. (d) The resulting $N_{\mathrm{AP}}(t / \tau)$ and $N_{\mathrm{AS}}(t / \tau)$ for one trial.
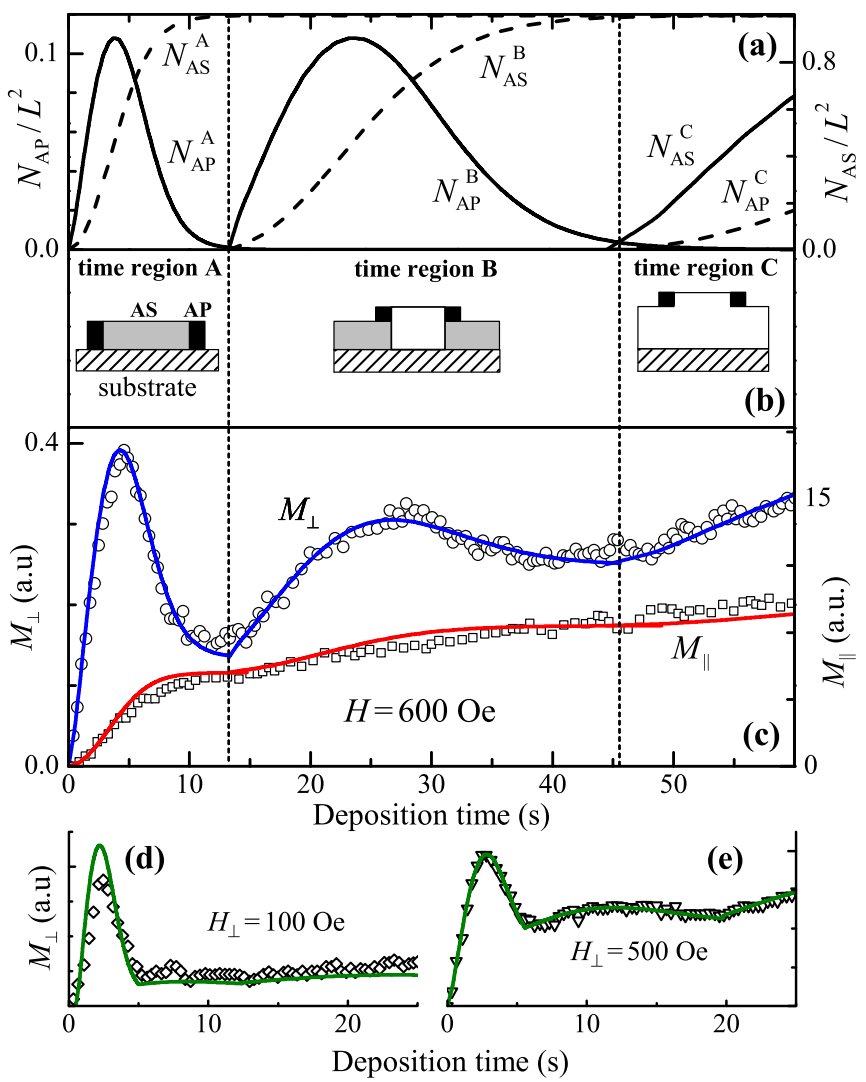

FIG. 3. (a) $N_{\mathrm{AP}}(t)$ and $N_{\mathrm{AS}}(t)$ for $L=300$ and 16 grains with $\epsilon=0.6$ and $L / 60$ wide rims employed in the calculations of both $M_{\|}(t)$ and $M_{\perp}(t)$ fitting curves shown in panel (c) for the first bilayer (denoted as A) and the subsequently grown third and fourth monolayers (B and $\mathrm{C}$, respectively) of a film grown using EC2 and $H=600 \mathrm{Oe} . N_{\mathrm{AP}}$ and $N_{\mathrm{AS}}$ of regions $\mathrm{B}$ and $\mathrm{C}$ differ from those in A by the greater time spans only; we also used $M_{s}=1400 \mathrm{emu} / \mathrm{cm}^{3}$, $K_{1}^{\text {hcp }}=-2.8 \times 10^{6} \mathrm{erg} / \mathrm{cm}^{3}$, and $K_{S}=-0.12 \mathrm{erg} / \mathrm{cm}^{2}$. Schematic pictures of the islands' growth modes are shown in (b) where black areas correspond to AP, gray to fcc AS, and white to hcp AS. Panels (d) and (e) present $M_{\perp}(t)$ data for films grown in $H=100$ and 500 Oe using EC1. Symbols and lines in (c)-(e) represent experiment and model, respectively. The curves in (d) and (e) were simulated employing the parameters used in (c) changing only the time spans and the $H$ value.

that should be straight lines, were built. The values for $n$ derived from their slopes are very close to 2 for all curves, as expected. Also, it is possible to nearly superimpose all differently simulated $N_{\mathrm{AP}}(t / \tau)$ curves by simple scaling, indicating that, if AP are responsible for the perpendicular MA, the later should not depend on the islands' shape and distribution, in agreement with experimental and theoretical results. ${ }^{7,18}$

Although the validity of such conventional KJMA calculations is certainly restricted, e.g., the growing particles may have different shapes and orientations, non-negligible incubation times may precede the onset of crystallization, etc., ${ }^{24}$ the shapes of the $N_{\mathrm{AP}}(t / \tau)$ and $N_{\mathrm{AS}}(t / \tau)$ curves should not vary qualitatively by removing the limitations of the KJMA theory.

Figure 3(c) shows $M_{\perp}(t)$ and $M_{\|}(t)$ data for $U=-1.3 \mathrm{~V}$ and $H=600$ Oe for films deposited using EC2 and the best fitting curve for the $\perp$ configuration. $N_{\mathrm{AP}}(t)$ and $N_{\mathrm{AS}}(t)$, used in the fittings of $M_{\perp}(t)$ employing $M_{s}=1400 \mathrm{emu} / \mathrm{cm}^{3}$ and $K_{1}^{\mathrm{hcp}}=-2.8 \times 10^{\frac{6}{6} \mathrm{erg} / \mathrm{cm}^{3}}$ (a value very close to that of Osgood et al. ${ }^{20}$ ) are plotted in panel (a). There, $N_{\mathrm{AP}}$ and $N_{\mathrm{AS}}$ of regions $\mathrm{B}$ and $\mathrm{C}$ differ from those of region $\mathrm{A}$ by the time 
spans only; panel (b) gives a schematic picture of the islands' growth.

There is an excellent agreement between model and experiment for the $\perp$ configuration. Due to the predominant $K_{D}$ term, $M_{\|}(t)$ does not depend on the other anisotropy parameters in regions B and C, see Eqs. (5) and (6). The model $M_{\|}(t)$ does not actually represent a fit to the experiment since it was calculated by using $N_{\mathrm{AP}}(t)$ and $N_{\mathrm{AS}}(t)$ from the $M_{\perp}(t)$ fitting; nevertheless, the coincidence with the experiment is quite good.

Due to the predominance of the perpendicular MA of the AP over their shape anisotropy (the latter is very weak at the initial stages of the deposition), we accepted that $\mathbf{m}_{\mathrm{AP}} \| H$ for $\mathbf{H}$ normal to the plane in region A. This is strongly supported by the very weak field dependence of the first peak, see Fig. 1(b), ascribed to the AS term in Eq. (1). The field-independent AP term in this expression, however, does not allow the estimation of $K_{\text {eff }}^{\mathrm{AP}}$ and one only knows that $\left|K_{1}^{\mathrm{AP}}\right|>K_{D}$; the lower limit of $\left|K_{1}^{\mathrm{AP}}\right|-K_{D}=4.0 \times 10^{6} \mathrm{erg} / \mathrm{cm}^{3}$ is obtained from the best fit of $M_{\|}(t)$ using Eq. (4).

$K_{1}^{\mathrm{AP}}$ was estimated by fittings $M_{\perp}(t)$ in both regions $\mathrm{B}$ and $\mathrm{C}$ using Eqs. (2) and (3). Here, differently to region A, the AP term depends on $H$ accounting for the gradual decrease of the second peak when decreasing $H$, as seen in Fig. 1(b). The effective anisotropy constant is positive giving $K_{D}>\left|K_{1}^{\mathrm{AP}}\right|$ so $K_{1}^{\mathrm{AP}}=-1.18 \times 10^{7} \mathrm{erg} / \mathrm{cm}^{3}$ when $K_{D}$ assumes its maximum value since there are 2 and 3 Co MLs already grown for $t$ varying in regions $\mathrm{B}$ and $\mathrm{C}$, respectively. Note that the estimated $K_{1}^{\mathrm{AP}}$ is approximately four times larger than that of bulk hcp Co.

$K_{S}=-0.12 \mathrm{erg} / \mathrm{cm}^{2}$ was estimated from $M_{\perp}(t)$ using $K_{\text {eff }}^{\mathrm{AS}, \mathrm{fcc}}=K_{D}+K_{S} / d, K_{\mathrm{eff}}^{\mathrm{AS}, \mathrm{hcp}}=K_{D}+K_{1}^{\mathrm{hcp}}+K_{S} / d$, and $K_{D}$ $=2 \pi M_{\mathrm{s}}^{2}$. Since both Co interfaces contribute to $K_{S}$, i.e., $K_{S}^{\mathrm{Co} / \text { solution }}$ and $K_{S}^{\mathrm{Au} / \mathrm{Co}}$ the former normally corresponding to easy-plane and the later to perpendicular MA, ${ }^{13}$ then $K_{S}^{\mathrm{Au} / \mathrm{Co}}$ is predominant.

We used identical procedures to reproduce both peaks. In principle, if the model describes the experiment correctly, one should also be able to detect a third peak in $M_{\perp}(t)$ during the growth of the fourth ML at high magnetic fields. However, due to the relatively small number of AP (there are 3 MLs already deposited), this peak falls into the noise level signal and cannot be distinguished.

The validity of our model is strongly supported by the agreement between experiment and simulation seen also in Figs. 3(d) and 3(e) for films grown using EC1. Here, we merely employed the model parameters from panel (c) changing appropriately the time spans only.

In summary, we have demonstrated that the two peaks in the perpendicular-to-the-plane magnetization versus deposition time variation of electrodeposited $\mathrm{Co} / \mathrm{Au}(111)$ layers could be solely explained by the exceptionally high magnetic anisotropy of the perimeter atoms of 2D nanoclusters formed during the growth. Although other factors such as strain relaxation at islands' coalescence, e.g., might also contribute to the appearance of the second peak, considering distinct anisotropy behavior of the islands' edges only ${ }^{6,7}$ gives a fairly good approximation when describing the experimental observations. Our results indicate that even at room temperature it is possible to sustain high anisotropy by increasing the islands' perimeter producing, e.g., islands with irregular shapes. Their size can be reduced much further than previously estimated without coming across the so-called superparamagnetic limit, thus opening excellent opportunities for materials engineering.

We are grateful to P. Allongue (CNRS, Ecole Polytechnique, France) because this work would have not been possible without his expertise with nanoscale electrodeposition on well defined $\mathrm{Au}(111)$ surfaces. This work has been supported by the Brazilian agency CNPq.

${ }^{1}$ A. Hernando, P. Crespo, M. A. García, E. Fernández Pinel, J. de la Venta, A. Fernández, and S. Penadés, Phys. Rev. B 74, 052403 (2006).

${ }^{2}$ L. Néel, J. Phys. Radium 15, 225 (1954).

${ }^{3}$ D. Sander, J. Phys.: Condens. Matter 16, R603 (2004).

${ }^{4}$ T. Koide, H. Miyauchi, J. Okamoto, T. Shidara, A. Fujimori, H. Fukutani, K. Amemiya, H. Takeshita, S. Yuasa, T. Katayama, and Y. Suzuki, Phys. Rev. Lett. 87, 257201 (2001).

${ }^{5}$ P. Gambardella, A. Dallmeyer, K. Maiti, M. C. Malagoli, W. Eberhardt, K. Kern, and C. Carbone, Nature 416, 301 (2002).

${ }^{6}$ P. Gambardella, S. Rusponi, M. Veronese, S. S. Dhesi, C. Grazioli, A. Dallmeyer, I. Cabria, R. Zeller, P. H. Dederichs, K. Kern, C. Carbone, and H. Brune, Science 300, 1130 (2003).

${ }^{7}$ S. Rusponi, T. Cren, N. Weiss, M. Epple, P. Buluschek, L. Claude, and H. Brune, Nature Mater. 2, 546 (2003).

${ }^{8}$ F. Luis, F. Bartolomé, F. Petroff, J. Bartolomé, L. M. García, C. Deranlot, H. Jaffrès, M. J. Martínez, P. Bencok, F. Wilhelm, A. Rogalev, and N. B. Brookes, Europhys. Lett. 76, 142 (2006); O. Šipr, S. Bornemann, J. Minár, S. Polesya, V. Popescu, A. Simůnek, and H. Ebert, J. Phys.: Condens. Matter 19, 096203 (2007).

${ }^{9}$ H. Brune and P. Gambardella, Surf. Sci. 603, 1812 (2009).

${ }^{10}$ A. Gündel, L. Cagnon, C. Gomes, A. Morrone, J. Schmidt, and P. Allongue, Phys. Chem. Chem. Phys. 3, 3330 (2001).

${ }^{11}$ P. Allongue, L. Cagnon, C. Gomes, A. Gündel, and V. Costa, Surf. Sci. 557, 41 (2004).

${ }^{12}$ A. Gündel, A. Morrone, J. Schmidt, L. Cagnon, and P. Allongue, J. Magn. Magn. Mater. 226-230, 1616 (2001).

${ }^{13}$ L. Cagnon, T. Devolder, R. Cortes, A. Morrone, C. Chappert, J. E. Schmidt, and P. Allongue, Phys. Rev. B 63, 104419 (2001).

${ }^{14}$ A. Gündel, T. Devolder, C. Chappert, J. E. Schmidt, R. Cortes, and P. Allongue, Phys. B 354, 282 (2004).

${ }^{15}$ B. Voigtländer, G. Meyer, and N. M. Amer, Phys. Rev. B 44, 10354 (1991); J. de la Figuera, J. E. Prieto, C. Ocal, and R. Miranda, ibid. 47, 13043 (1993).

${ }^{16} \mathrm{P}$. Allongue, H. Brune, and H. Gerischer, Surf. Sci. 275, 414 (1992).

${ }^{17}$ A. Gündel, Ph.D. thesis, UFRGS, Porto Alegre, RS, 2002.

${ }^{18}$ E. Y. Vedmedenko, H. P. Oepen, and J. Kirschner, J. Magn. Magn. Mater. 256, 237 (2003)

${ }^{19}$ A. N. Bogdanov, U. K. Rößler, and K.-H. Müller, J. Magn. Magn. Mater. 242-245, 594 (2002).

${ }^{20}$ R. M. Osgood III, K. T. Riggs, and A. E. Johanson, Phys. Rev. B 56, 2627 (1997).

${ }^{21}$ A. N. Kolmogorov, Bull. Acad. Sci. USSR Math. Ser. 1, 355 (1937).

${ }^{22}$ W. A. Johnson and R. F. Mehl, Trans. Am. Inst. Min., Metall. Pet. Eng. 135, 416 (1939).

${ }^{23}$ M. Avrami, J. Chem. Phys. 7, 1103 (1939); M. Avrami, J. Chem. Phys. 8, 212 (1940); M. Avrami, J. Chem. Phys. 9, 177 (1941).

${ }^{24}$ T. Pusztai and L. Gránásy, Phys. Rev. B 57, 14110 (1998). 